\title{
Cronotipo, Qualidade do sono e Rendimento escolar em adolescentes - uma revisão
}

\section{da literatura}

\author{
Chronotype, Sleep Quality and School Performance in adolescents- an integrative literature review \\ Cronotipo, Calidad del Sueño y Desempeño Escolar em adolescentes - revisión integrativa de la
}

literatura

Recebido: 02/09/2021 | Revisado: 08/09/2021 | Aceito: 09/09/2021 | Publicado: 11/09/2021

\author{
Gabriele Lima de Araújo \\ ORCID: https://orcid.org/0000-0002-7343-7567 \\ Universidade de Pernambuco, Brasil \\ E-mail: gabriele.lima@hotmail.com \\ Taiana Jéssica Oliveira Araújo \\ ORCID: https://orcid.org/0000-0002-0581-8907 \\ Universidade de Pernambuco, Brasil \\ E-mail: taianaoliveiraa14@gmail.com \\ Mariana Araújo Coutinho da Silveira \\ ORCID: https://orcid.org/0000-0003-3365-5858 \\ Universidade de Pernambuco, Brasil \\ E-mail: marianaaraujocs@gmail.com \\ Thayane Maria Botelho Florêncio \\ ORCID: https://orcid.org/0000-0002-9864-3631 \\ Universidade de Pernambuco, Brasil \\ E-mail: thayane.mbf@gmail.com \\ Mônica Vilela Heimer \\ ORCID: https://orcid.org/0000-0003-3842-192X \\ Universidade de Pernambuco, Brasil \\ E-mail: monica.vilelaheimer@gmail.com
}

\begin{abstract}
Resumo
Este trabalho teve como objetivo investigar a associação do cronotipo com a qualidade do sono e rendimento escolar dos adolescentes.Realizou-se uma Revisão Integrativa onde a mesma foi conduzida de acordo com as informações coletadas nas bases Pubmed, Scopus e Embase seguindo as diretrizes do PRISMA. Foram incluídos artigos observacionais, nos idiomas inglês, português e espanhol, sem restrição de período de publicação, abrangendo a faixa etária de 10 a 19 anos e que respondiam a pergunta condutora: "Existe associação do cronotipo com a qualidade do sono e o rendimento escolar em adolescentes?" Foram utilizados os descritores "chronotype", "learning", "Adolescent e seus sinônimos reconhecidos pelo vocabulário Mesch e Desc. Foram encontrados 2625 artigos, dos quais 2220 foram selecionados para a leitura de títulos e resumos. Após esta etapa 16 foram selecionados para a leitura na íntegra, sendo selecionados 5 artigos. Em seguida foi realizada uma busca manual na referência desses artigos, resultando nainclusão de mais 8 artigos, totalizando 13 artigos para compor a síntese qualitativa desta revisão. Verificou-se que cronotipos vespertinos apresentam mais distúrbios relacionados ao sono, problemas emocionais e mau desempenho escolar. Verificou-se uma associação do cronotipo com a qualidade do sono e o rendimento escolar em adolescentes. Os adolescentes com cronotipo vespertino demostraram má qualidade do sono, menores níveis de atenção e desempenho escolar e maior risco de desenvolvimento de problemas comportamentais.
\end{abstract}

Palavras-chave: Qualidade do sono; Cronotipo; Aprendizagem; Adolescentes.

\section{Abstract}

This study aimed to investigate the association of chronotype with sleep quality and school performance of adolescents. An Integrative Review was carried out in which it was conducted according to the information collected in the Pubmed, Scopus and Embase databases, following the PRISMA guidelines . Observational articles were included, in English, Portuguese and Spanish, with no restriction on the period of publication, covering the age group from 10 to 19 years old and answering the leading question: "Is there an association of chronotype with sleep quality and school performance in teenagers?" The descriptors "chronotype", "learning", "Adolescent and its synonyms recognized by the vocabulary Mesch and Desc were used. 2625 articles were found, of which 2220 were selected for reading titles and abstracts. After this step 16 were selected for full reading, 5 articles were selected. Then, a manual search was performed in the reference of these articles, resulting in the inclusion of 8 more articles, totaling 13 articles to compose the qualitative synthesis of this review. It was found that afternoon chronotypes have more sleep-related disorders, emotional problems and poor 
school performance. There was an association of chronotype with sleep quality and school performance in adolescents. Adolescents with afternoon chronotype showed poor sleep quality, lower levels of attention and school performance, and greater risk of developing behavioral problems.

Keywords: Sleep quality; Chronotype; Learning; Teenagers.

\section{Resumen}

Este estudio tuvo como objetivo investigar la asociación del cronotipo con la calidad del sueño y el rendimento escolar de los adolescentes, se realizó una Revisión Integrativa en la que se realizó de acuerdo con la información recolectada en las bases de datos Pubmed, Scopus y Embase, siguiendo las pautas PRISMA. Se incluyeron artículos observacionales, en inglés, portugués y español, sin restricción en el período de publicación, cubriendo el grupo de edad de 10 a 19 años y respondiendo a la pregunta principal: “¿Existe una asociación del cronotipo con la calidad del sueño y el rendimiento escolar? en los adolescentes? Se utilizaron los descriptores "cronotipo", "aprendizaje", "Adolescente y sus sinónimos reconocidos por el vocabulario Mesch y Desc". Se encontraron 2625 artículos, de los cuales 2220 fueron seleccionados para lectura de títulos y resúmenes. Después de seleccionar este paso 16 para su lectura completa, se seleccionaron 5 artículos. Luego, se realizó una búsqueda manual en la referencia de estos artículos, resultando en la inclusión de 8 artículos más, totalizando 13 artículos para componer la síntesis cualitativa de esta revisión. Se encontró que los cronotipos vespertinos tienen más trastornos relacionados con el sueño, problemas emocionales y bajo rendimiento escolar. Hubo asociación del cronotipo con la calidad del sueño y el rendimiento escolar en adolescentes. Los adolescentes con cronotipo vespertino presentaron mala calidad del sueño, menores niveles de atención y rendimiento escolar y mayor riesgo de desarrollar problemas de conducta.

Palabras clave: Calidad del sueño; Cronotipo; Aprendiendo; Adolescentes.

\section{Introdução}

O sono é uma ação reversível de desligamento da percepção ao ambiente, essencial para a manutenção da saúde física e cognitiva. Esse comportamento é mediado pela ação de uma série de componentes, como os hormônios, e seus picos de liberação e estão associados a ritmos circadianos (Oliveira, Silva, Alves, Silva \& Pinto, 2019).

O sono é fator essencial para a saúde e bem-estar físico e mental (Hairston \& Shpitalni 2016). Existem múltiplas dimensões de sono, incluindo a duração suficiente, regulação dos padrões de sono-vigília, eficiência e satisfação (Bluden \& Galland, 2014). Sono insuficiente (menos de 8 horas) e padrões irregulares de sono-vigília estão associados com humor deprimido, comportamentos de risco, risco do surgimento de doenças cardiovasculares e obesidade (Malone et al., 2015).

Fatores como sexo, idade e região também podem influenciar no tempo diário de sono, além de outros fatores biológicos, como o cronotipo, que afeta diretamente na adaptação do indivíduo ao ambiente, comprometendo sua atenção no decorrer do dia (Batista et al., 2018).

O cronotipo classifica o indivíduo de acordo com a sua predisposição, biologicamente determinada, para desenvolver atividades de forma otimizada em momentos específicos do dia (Figueiredo, Hipolito \& Tomas, 2018). Os indivíduos podem ser classificados em matutinos, que são os que preferem acordar e dormir cedo, apresentando bom nível de alerta e desempenho nas atividades durante a manhã e os vespertinos, que tendem a acordar e dormir tarde, apresentando melhor desempenho nas atividades durante a tarde ou à noite. Já os indiferentes são os que não têm preferência específica.

No início da adolescência, o ritmo circadiano tende a sofrer mudanças, onde cronotipos vespertinos e norturnos tornamse mais prevalentes. É importante considerar o cronotipo nas atividades diárias do adolescente, visto que estudar em horários condizentes com a preferência fisiológica destes, propiciará uma maior absorção do assunto e consequentemente um melhor rendimento escolar (Mello, Mello \& Mello, 2018).

Vale destacar que a adolescência é caracterizada por importantes mudanças biopsicossociais e emocionais, até mesmo quando se trata do padrão do ciclo vigília-sono, visto que ocorrem importantes mudanças no padrão do ciclo vigília, como o atraso na fase do sono, em que os adolescentes tendem a ficar até mais tarde acordados, no entanto, atividades sociais diárias deste grupo populacional os abrigam a acordar cedo, consequentemente, os adolescentes tendem a apresentar sonolência diurna (Martins, 2018). O padrão irregular e os distúrbios do sono nessa faixa etária são justificados por uma junção de influências dos 
fatores biológicos, psicológicos e socioculturais, associados às mudanças no ritmo circadiano, maior autonomia para escolher os horários de sono, pressão acadêmica, além do uso de dispositivos de tela e rede social. (Batista et al., 2018; Felden et al., 2016). Assim, rotinas vespertinas, atraso para o início do sono e a sonolência diurna dificultam a manutenção do estado de alerta e, consequentemente, a adaptação aos horários das atividades diárias sociais, como a escola. Por isso é importante compreender os comportamentos e ritmos biológicos no ambiente escolar, uma vez que estes podem afetar a memória, a cognição e a aprendizagem (Meyer et al., 2017; Adelantado-Renau, Diez-Dernandez, Beltran-Valls, Soriano-Maldonado \& Moliner-Urdiale, 2019).

Diante do exposto, o presente estudo tem como objetivo avaliar o cronotipo e a sua associação com a qualidade do sono e o rendimento escolar em adolescentes. Para tanto optou-se por realizar um estudo de revisão integrativa como estratégia de pesquisa para identificar as evidências existentes na literatura a respeito deste tema.

\section{Metodologia}

Trata-se de uma revisão integrativa da literatura, baseada na seguinte pergunta norteadora: "Existe associação do cronotipo com a qualidade do sono e o rendimento escolar em adolescentes?"

A revisão foi construída por meio da análise de publicações de artigos nas bases de dados sobre a temática "cronotipo e aprendizagem" disponíveis na literatura internacional e nacional indexados no National Library of Medicine (Pubmed), SciVerse Scopus (Scopus) e Embase.

Para o refinamento adequado da pesquisa foram definidos como critérios de inclusão os artigos nos idiomas inglês, português e espanhol, com os resumos disponíveis nas bases de dados supracitadas, que abordassem o tema Cronotipo e Aprendizagem, na faixa etária de 10 a 19 anos, caracterizando a adolescência segundo a Organização Mundial de Saúde (OMS).

Excluiu-se desta seleção os estudos que se encontravam repetidos nas bases de dados, os estudos que se classificassem como artigo de revisão de literatura e os que não se apresentavam em formato de artigo, como guidelines, cartas, editoriais, teses e dissertações.

Foi realizada uma revisão por pares, ou seja, os artigos foram analisados por dois pesquisadores individualmente (G.L.A e M.A.C.S.) para posterior análise em conjunto, seguindo os critérios de inclusão e exclusão. Caso houvesse alguma divergência um terceiro revisor foi consultado (M.V.H.) até obter-se um consenso, a fim de trazer maior confiabilidade para a seleção dos estudos da síntese.

Dessa forma, os artigos passaram pela etapa de leitura de títulos e resumos. Aqueles que possuíam títulos e resumos que não contemplavam o assunto pesquisado foram excluídos. Já os estudos que preencheram os critérios de inclusão foram selecionados para leitura do texto completo, resultando na seleção dos artigos incluídos nesta síntese (Figura 1).

\section{Resultados}

Um total de 2625 artigos foram encontrados nas bases de dados pesquisadas. Foram excluídos 405 artigos duplicados e 2204 após leitura de títulos e resumos, resultando em 16 artigos para a leitura na íntegra, sendo selecionados 5 artigos. Após esta etapa foi realizado uma busca manual nas referências desses artigos, resultando na inclusão de mais 8 artigos, totalizando 13 artigos para compor a síntese qualitativa desta revisão.

O processo de identificação, inclusão e exclusão dos estudos é apresentado na Figura 1. 
Figura 1 - Fluxograma da seleção de artigos seguindo as orientações do PRISMA.

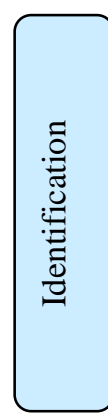

Registros identificados nas bases de dados: Scopus (99);

Embase (1718); Pubmed (808)

Total $=2625$
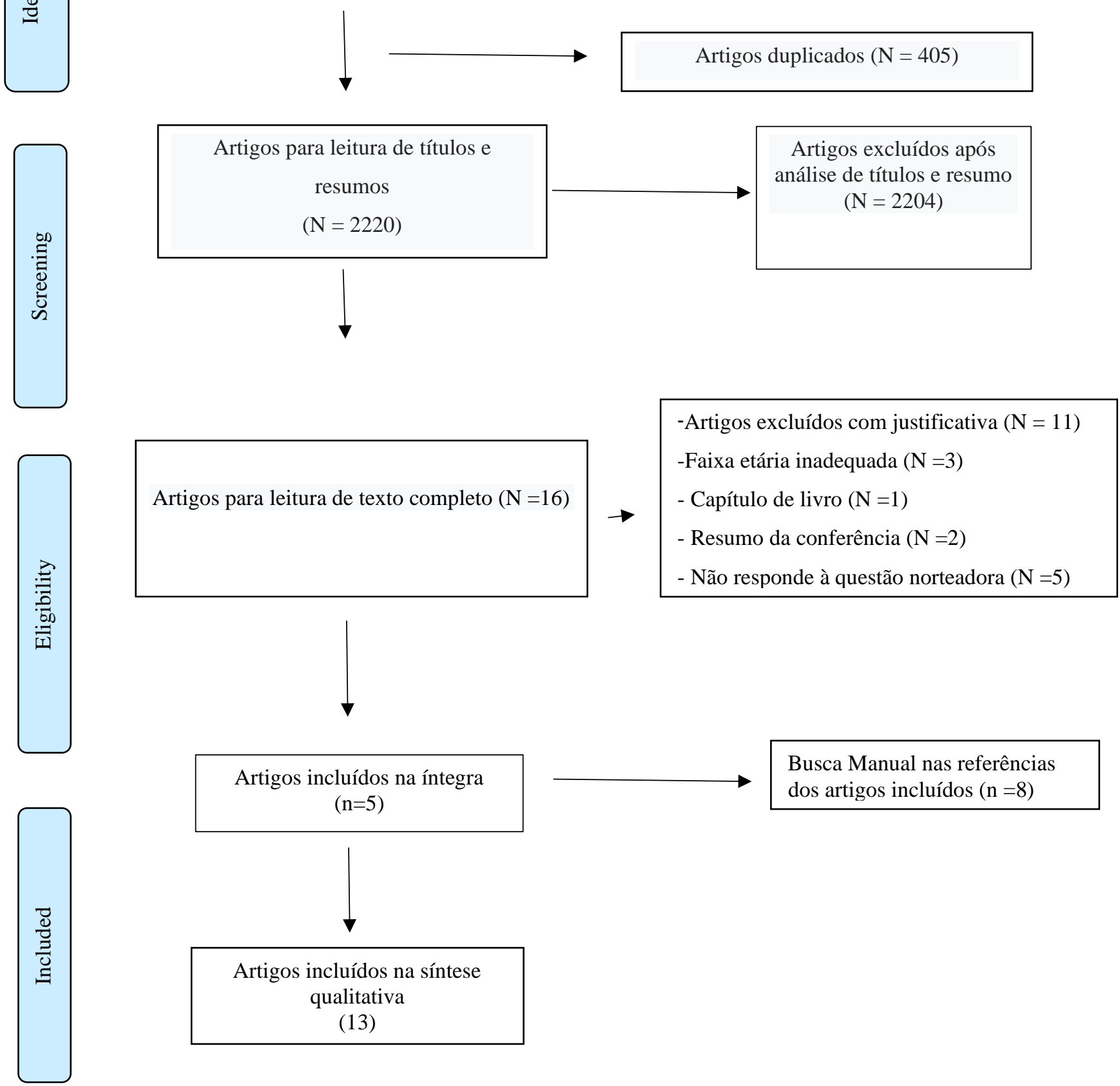

Fonte: Autores.

\section{Características dos estudos}

Quanto à distribuição temporal, 7,7\% dos estudos foram realizados em 1998 (Epstein, Chillag \& Lavie); 15,3\% em 2010 (Clarisse, Le Floc'h, Kindelberger \& Feunteun; Borisenkov, Perminova \& Kosova) e 2012 (Escribano, Díaz-Morales, Delgado \& Collado; Hahn et al.), 30,8\% em 2013 (Vollmer, Potsch \& Randler; Roeser, Scharb \& Kubler; Preckel et al. ; DíazMorales \& Escribano ), 7,7 \% em 2014 (Escribano \& Díaz-Morales), 15,3\% em 2015 (van der Vinne et al.; Rahafar et al.) e 
7,7\% em 2017 (Zerbini et. al.). Em relação a distribuição geográfica, 7,7 \% das pesquisas foram realizadas no continente americano (HAHN et al); 23\% na Ásia (Epstein et al.; Borisenkov et al; Rahafar Et al.); e 69,2\% na Europa (Zerbini et. al.; Vinne et al.; Escribano \& Díaz-Morales; Díaz- Morales \& Escribano; Preckel et al; Roeser, Scharb \& Kubler; Vollmer et al.; Escribano et al.; Clarisse et al). Quanto ao desenho do estudo, $100 \%$ dos artigos foram transversais (Zerbini et. al.; van der Vinne et al.; Escribano \& Díaz-Morales; Díaz- Morales \& Escribano; Preckel et al; Roeser et al.; Vollmer et al.; Escribano et al.; Clarisse et al.; Hahn et al.; (Epstein et al.; Borisenkov et al.; Rahafar Et al)

Quanto à definição do cronotipo, todos os artigos usaram ferramentas validadas (Zerbini et. al.; van der Vinne et al.; Escribano \& Díaz-Morales; Díaz- Morales \& Escribano; Preckel et al; Roeser et al.; Vollmer et al.; Escribano et al.; Clarisse et al.; Hahn et al.; (Epstein et al.; Borisenkov et al.; Rahafar et al). Em relação à análise da qualidade do sono, 69,2\% dos estudos incluídos nesta síntese utilizaram instrumentos já validados (Zerbini et. al.; van der Vinne et al.; Díaz- Morales \& Escribano; Preckel et al; Roeser et al.; Vollmer et al.; Clarisse et al.; Hahn et al.; Rahafar et al.) 30,8\% dos trabalhos utilizaram a média descrita pelos participantes (Epstein et al.; Borisenkov et al.; Escribano et al.; Escribano \& Díaz-Morales)

Em relação ao rendimento escolar e aos problemas comportamentais, $23 \%$ das pesquisas utilizam instrumentos validados para avaliação (Hahn et al.; Escribano \& Díaz-Morales; Rahafar et al) 15,3\% utilizaram o relato dos próprios adolescentes quanto as variáveis citadas (Epstein et al.; Escribano et al.) e 61,6\%, o relato e a observação por meio da análise de notas e atividades acadêmicas (Zerbini et. al.; van der Vinne et al.; Díaz- Morales \& Escribano; Preckel et al; Roeser et al.; Vollmer et al.; Clarisse et al.; Borisenkov et al.)

No tocante à associação entre as variáveis estudadas, 46,1\% dos estudos evidenciaram desempenho escolar inferior e notas mais baixas em cronotipos vespertinos (Zerbini et. al.; van der Vinne et al.; Díaz- Morales \& Escribano; Preckel et al; Vollmer et al.; Escribano \& Díaz-Morales). O cronotipo também foi associado a baixa duração de sono em 15,3\% dos estudos, onde os cronotipos vespertinos apresentaram déficit de sono comparado com os demais cronotipos (Escribano \& Díaz-Morales; Zerbini et al.), e a presença de sonolência diurna em outros 15,3\% dos estudos (Roeser et al.; Epstein et al.). Por sua vez, a duração do sono foi associada ao rendimento escolar em 38,5\% dos estudos (Escribano \& Díaz-Morales; Borisenkov et al.; DíazMorales \& Escribano; van der Vinne et al.; Zerbine et al), no qual os que apresentavam sono mais longo obtiveram melhor rendimento.

Em 23\% dos estudos o período de realização das provas escolares teve um efeito significativo no desempenho dos adolescentes (Clarisse et al.; van der Vinne et al.; Hahn et al), onde os adolescentes que realizaram as provas respeitando o seu cronotipo, ou seja, em seu período ideal de realização de atividades, obtiveram melhor desempenho.

Quanto ao nível de atenção e consciência, 15,3 \% dos estudos associaram o nível de consciência ao cronotipo (Preckel et al.; Rahafar et al), onde os matutinos pareciam estar mais conscientes e ativos do que os demais. Já com relação ao nível de atenção e o cronotipo, 25\% dos estudos identificaram associação entre as variáveis, em que os matutinos demostravam melhores níveis de atenção no período escolar (Vollmer et al.; Epstein et al.; Escribano \& Díaz-Morales).

O Quadro 1 apresenta uma visão panorâmica dos estudos incluídos nesta revisão destacando: autor/ano/país de publicação, desenho de estudo, amostra, faixa etária, instrumento utilizado, resultados e conclusão. 
Quadro 1 - Disposição dos dados de acordo com autor/ano/país de publicação, desenho de estudo, amostra, faixa etária e instrumento utilizados, resultados e conclusão.

\begin{tabular}{|c|c|c|c|c|c|c|}
\hline $\begin{array}{l}\text { Autor } \\
\text { Ano } \\
\text { País }\end{array}$ & $\begin{array}{l}\text { Desenho } \\
\text { de } \\
\text { estudo }\end{array}$ & Amostra & $\begin{array}{l}\text { Faixa } \\
\text { etária } \\
(\text { anos })\end{array}$ & Instrumentos & Resultados & Conclusão \\
\hline $\begin{array}{c}\text { Zerbini } \\
\text { Et. al } \\
2017 \\
\text { Holanda }\end{array}$ & Transversal & 426 & $11-16$ & $\begin{array}{l}\text { Número médio de notas } \\
\text { por aluno; } \\
\text { Munich Questionário } \\
\text { ChronoType (MCTQ) + } \\
\text { duração do sono } \\
\text { Frequência escolar: } \\
\text { sistema de registro da } \\
\text { escola }\end{array}$ & $\begin{array}{l}\text { Os cronotipos vepertinos } \\
\text { obtiveram notas mais baixas } \\
\text { em comparação com os } \\
\text { cronotipos matutinos (p = } \\
0,0212 \text { ) } \\
\text { O cronótipo vespertino foi } \\
\text { relacionado à frequência } \\
\text { escolar e a probabilidade de } \\
\text { chegar atrasado à primeira } \\
\text { aula do dia }(\mathrm{p}=0,0106) \text {. } \\
\text { A curta duração do sono foi } \\
\text { significativamente associada a } \\
\text { notas mais baixas e a } \\
\text { cronotipos vespertino (p = } \\
0,0034)\end{array}$ & $\begin{array}{l}\text { O cronotipo foi } \\
\text { associado a qualidade do } \\
\text { sono e ao rendimento } \\
\text { escolar }\end{array}$ \\
\hline $\begin{array}{c}\text { Rahafar } \\
\text { Et al. } \\
2015 \\
\text { Irã }\end{array}$ & Transversal & 158 & 17,5 & $\begin{array}{l}\text { GPAs -registradas pelo } \\
\text { ministério da educação. } \\
\text { Questionário matinal } \\
\text { reduzido - noturno(rME } \\
\text { Q) } \\
\text { Estoque NEO de cinco } \\
\text { fatores (NEO - FFI) } \\
\text { Teste de inventário de } \\
\text { ansiedade }\end{array}$ & $\begin{array}{l}\text { Os adolescentes matutinos } \\
\text { demonstraram estar mais } \\
\text { conscientes e ativos do que os } \\
\text { vespertinos }(p<0,001) \text { e os } \\
\text { intermediários }(p=0,009) \text {, } \\
\text { Cronotipos vespertinos } \\
\text { tendiam a apresentar mais } \\
\text { ansiedade, porém não houve } \\
\text { diferença estatística ( } \mathrm{p}=0.087)\end{array}$ & $\begin{array}{l}\text { O cronótipo foi um } \\
\text { significativo preditor de } \\
\text { rendimento escolar } \\
\text { quando foi moderado } \\
\text { pela conscientização, } \\
\text { mas apenas em meninas. } \\
\text { O cronotipo não } \\
\text { apresentou influência } \\
\text { significativa em relação } \\
\text { a ansiedade. }\end{array}$ \\
\hline $\begin{array}{l}\text { Van der } \\
\text { Vinne } \\
\text { Et al. } \\
2015 \\
\text { Holanda }\end{array}$ & Transversal & 741 & $11-18$ & $\begin{array}{ll}\text { Notas das disciplinas } \\
\text { Questionário } & \text { do } \\
\text { ChronoType } & \text { de } \\
\text { Munique } & \end{array}$ & $\begin{array}{l}\text { Cronotipos vespertinos } \\
\text { tiveram notas } \\
\text { significantemente mais baixas } \\
\text { do que os matutinos ( } p= \\
0,0042) \\
\text { Redução do tempo de sono nos } \\
\text { dias escolares foi associado a } \\
\text { notas mais baixas }(p=0,0011) \\
\text { - As médias as notas variaram } \\
\text { significativamente com o } \\
\text { horário escolar, as notas das } \\
\text { provas foram inferiores no } \\
\text { período inicial do dia } \\
(p<0,0001)\end{array}$ & $\begin{array}{l}\text { O cronotipo, a idade e o } \\
\text { horário } r \text { escolar } \\
\text { demostraram influência } \\
\text { significativa em relação } \\
\text { ao tempo de sono e } \\
\text { medias das notas } \\
\text { escolares, onde } r \text { os } \\
\text { vespertinos rra } \\
\text { adolescentes } \\
\text { velhos, apresentaram } \\
\text { notas mais baixas e } \\
\text { tempo de sono reduzido. }\end{array}$ \\
\hline $\begin{array}{c}\text { Escribano } \\
\text { \& Díaz- } \\
\text { Morales } \\
2014 \\
\text { Espanha }\end{array}$ & Transversal & 669 & $12-16$ & $\begin{array}{l}\text { Cronotipo } \\
\text { Morningness/Eveningne } \\
\text { ss Scale for Children } \\
\text { MESC } \\
\text { Teste do Quadrado de } \\
\text { Letras (SLT) } \\
\text { Subteste de raciocínio } \\
\text { indutivo } \\
\text { Duração do sono: auto } \\
\text { relatado }\end{array}$ & $\begin{array}{l}\text { A idade obteve uma correlação } \\
\text { negativa quanto ao cronotipo e } \\
\text { duração do sono . } \\
\text { Cronotipo apresentou } \\
\text { correlação positiva com à } \\
\text { duraça do sono, onde } \\
\text { cronotipo vespertinos } \\
\text { apresentaram sono reduzido. } \\
\text { Em geral, os meninos } \\
\text { matutinos alcançaram níveis } \\
\text { mais elevados de atenção do } \\
\text { que as meninas matutinas. }\end{array}$ & $\begin{array}{l}\text { A atenção aumentou ao } \\
\text { longo do dia escolar em } \\
\text { ambos os cronótipo. Os } \\
\text { meninos tendem a } \\
\text { alcançar em média e em } \\
\text { diferentes momentos do } \\
\text { dia (no meio e no final } \\
\text { do dia) maior atenção do } \\
\text { que as meninas }\end{array}$ \\
\hline
\end{tabular}




\begin{tabular}{|c|c|c|c|c|c|c|}
\hline $\begin{array}{l}\text { Díaz- } \\
\text { Morales } \\
\quad \& \\
\text { Escribano } \\
2013 \\
\text { Espanha }\end{array}$ & Transversal & 887 & $12-16$ & $\begin{array}{l}\text { Cronotipo } \\
\text { Morningness/Eveningne } \\
\text { ss Scale for Children } \\
\text { MESC } \\
\text { School Sleep Habits } \\
\text { Survey } \\
\text { Média de notas (GPA) }\end{array}$ & 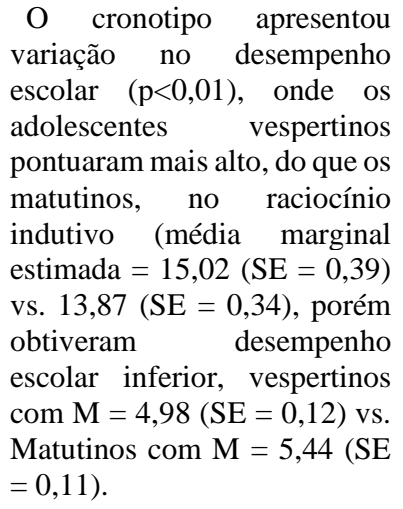 & $\begin{array}{l}\text { Adolescentes com } \\
\text { cronotipo matutino, que } \\
\text { estudam no horário } \\
\text { matinal, apresentaram } \\
\text { desempenho escolar } \\
\text { superior. }\end{array}$ \\
\hline $\begin{array}{c}\text { Preckel et } \\
\text { al } \\
2013 \\
\text { Alemanha }\end{array}$ & Transversal & 272 & 15,6 & 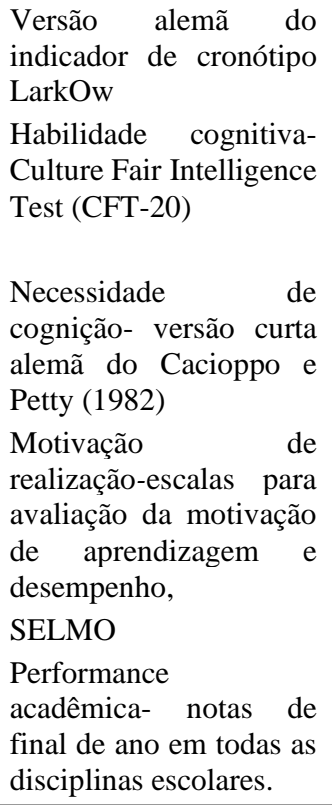 & $\begin{array}{l}\text { O cronotipo vespertino } \\
\text { mostrou correlações negativas } \\
\text { com a consciência, } \\
\text { orientações de metas de } \\
\text { desempenho e uma correlação } \\
\text { positiva com evasão escolar } \\
(\mathrm{p}<0.05) \text {. }\end{array}$ & $\begin{array}{l}\text { O cronotipo demostrou } \\
\text { estar relacionado ao } \\
\text { desempenho acadêmico. } \\
\text { Os matutinos tiveram } \\
\text { uma relação positiva } \\
\text { com o desempenho } \\
\text { acadêmico, enquanto } \\
\text { que os vespertinos, se } \\
\text { relacionam } \\
\text { negativamente com o } \\
\text { desempenho acadêmico }\end{array}$ \\
\hline $\begin{array}{c}\text { Roeser et } \\
\text { al. } \\
2013 \\
\text { Alemanha }\end{array}$ & Transversal & 273 & $14-16$ & 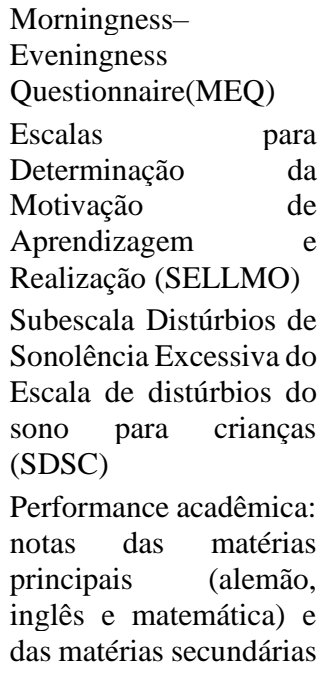 & $\begin{array}{l}\text { Cronotipo e aprendizagem } \\
\text { foram associados }(\mathrm{p}=0,009) \text {; } \\
\text { assim como a aprendizagem } \\
(\mathrm{p}<0,001) \text { se correlacionou } \\
\text { positivamente com } \\
\text { desempenho acadêmico. } \\
\text { Os cronotipos vespertinos } \\
\text { relataram mais sonolência } \\
\text { diurna do que os matutinos ( } \\
=0,003) \text {. }\end{array}$ & $\begin{array}{l}\text { Os cronotipos } \\
\text { vespertinos tenderam a } \\
\text { apresentar maior risco } \\
\text { de sonolência diurna. Os } \\
\text { tipos Matutinos } \\
\text { apresentaram melhor } \\
\text { desempenho em relação } \\
\text { aos objetivos de } \\
\text { aprendizagem do que os } \\
\text { vespertinos, }\end{array}$ \\
\hline $\begin{array}{l}\text { Vollmer } \\
\text { et al. } \\
2013 \\
\text { Alemanha }\end{array}$ & Transversal & 1977 & 13,38 & $\begin{array}{l}\text { Escala composta de } \\
\text { maturidade (CSM) } \\
\text { Avaliação } \\
\text { desempenho do } \\
\text { (Notas,Atenção) }\end{array}$ & $\begin{array}{l}\text { Os tipos matutinos obtiveram } \\
\text { uma correlação positiva com } \\
\text { maiores notas e nível de } \\
\text { atenção }(\mathrm{R} 2=0,007) \text {, em } \\
\text { relação aos cronotipos } \\
\text { vespertinos. } \\
\text { compartilhando melhores } \\
\text { classificações }(\mathrm{R} 2=0,052) \text { e } \\
\text { superior } \\
\text { atenção }(\mathrm{R} 2=0,007) .\end{array}$ & $\begin{array}{l}\text { O cronótipo foi um } \\
\text { preditor significativo de } \\
\text { notas, bem como de } \\
\text { desempenho no teste de } \\
\text { atenção, com tipos } \\
\text { matutinos obtendo } \\
\text { melhores notas e maior } \\
\text { atenção. }\end{array}$ \\
\hline
\end{tabular}




\begin{tabular}{|c|c|c|c|c|c|c|}
\hline $\begin{array}{l}\text { Hahn } \\
\text { et al } \\
2012 \\
\text { EUA }\end{array}$ & Transversal & 80 & $11-14$ & $\begin{array}{l}\text { Escala de preferências } \\
\text { matutinas-noturnas } \\
\text { infantis (CMEP) } \\
\text { Tarefa Go-Nogo } \\
\text { Tarefa de Apontamento } \\
\text { Auto ordenado (SOP) } \\
\text { Tarefa de mudança intra } \\
\text { e extradimensional (ID / } \\
\text { ED) } \\
\text { TheIowa Gambling } \\
\text { Task }\end{array}$ & $\begin{array}{l}\text { O cronótipo influenciou no } \\
\text { desempenho das tarefas de } \\
\text { acordo com o horário de } \\
\text { aplicação, onde os vespertinos } \\
\text { apresentaram melhor } \\
\text { desempenho quando testados } \\
\text { no período noturno e os } \\
\text { matutinos no período da } \\
\text { manhã (p=0,005) }\end{array}$ & $\begin{array}{l}\text { Os participantes testados } \\
\text { respeitando o seu } \\
\text { cronotipo, pobtiveram } \\
\text { melhor desempenho nas } \\
\text { atividades. }\end{array}$ \\
\hline $\begin{array}{l}\text { Escribano } \\
\text { Et al } \\
2012 \\
\text { Espanha }\end{array}$ & Transversal & 1133 & $12-16$ & $\begin{array}{l}\text { Cronotipo } \\
\text { Morningness/Eveningne } \\
\text { ss Scale for Children } \\
\text { MESC } \\
\text { Desempenho escolar: } \\
\text { Nível subjetivo de } \\
\text { realização, notas auto } \\
\text { relatadas. } \\
\text { Hábitos de sono: auto } \\
\text { relatado }\end{array}$ & $\begin{array}{l}\text { Os vespertinos relataram notas } \\
\text { mais baixas que os matutinos. } \\
(p=0,018) \\
\text { A redução do desempenho } \\
\text { escolar e o tempo total de sono } \\
\text { foi associado com a idade de } \\
\text { acordo com o cronotipo. } \\
(p=0.005)\end{array}$ & $\begin{array}{l}\text { Cronotipos vespertinos } \\
\text { obtiveram } \\
\text { pior desempenho } \\
\text { escolar, diminuição do } \\
\text { tempo de sono } \\
\text { em comparação r com } \\
\begin{array}{lr}\text { Matutinos } & \text { e } \\
\text { intermediários. }\end{array}\end{array}$ \\
\hline $\begin{array}{l}\text { Borisenko } \\
\text { v } \\
\text { Et al. } \\
2010 \\
\text { Rússia }\end{array}$ & Transversal & 1101 & 16,1 & $\begin{array}{l}\text { O questionário do } \\
\text { cronótipo de Munique } \\
\text { (MCTQ) } \\
\text { Duração do sono = } \\
\text { duração do sono nos dias } \\
\text { da semana x } 6+\text { duração } \\
\text { do sono nos fins de } \\
\text { semana/7. } \\
\text { Rendimento: notas } \\
\text { escolares }\end{array}$ & $\begin{array}{l}\text { O cronótipo exerceu um efeito } \\
\text { aproximadamente } 2 \text { vezes } \\
\text { maior do que a duração do } \\
\text { sono no desempenho escolar. } \\
(\mathrm{p}<0,00001) \\
\text { Houve um atraso de fase de do } \\
\text { sono entre as idades de } 11 \text { e } 22 \\
\text { anos. }\end{array}$ & $\begin{array}{l}\text { Dessincronização do } \\
\text { ritmo circadiano, } \\
\text { Influenciou no } \\
\text { aproveitamento escolar } \\
\text { de crianças e estudantes } \\
\text { universitários que } \\
\text { apresentaram sono } \\
\text { inadequado, onde os } \\
\text { cronótipo vespertinos } \\
\text { apresentaram pior } \\
\text { desempenho em relação } \\
\text { ao matutino e } \\
\text { intermediário. }\end{array}$ \\
\hline $\begin{array}{l}\text { Clarisse } \\
\text { et al } \\
2010 \\
\text { França }\end{array}$ & Transversal & 17 & 16,5 & $\begin{array}{l}\text { Versão adaptada de } \\
\text { Horne e Östberg } \\
\text { Questionáriomatinal- } \\
\text { verpertino (MEQ) } \\
\text { Teste de cruzamento de } \\
\text { números para mesurar a } \\
\text { atenção. }\end{array}$ & $\begin{array}{l}\text { - As pontuaçoses e desempenho } \\
\text { dos participantes do tipo } \\
\text { matutino se mantiveram } \\
\text { estáveis ao longo do dia, } \\
\text { diferente dos vespertinos que } \\
\text { apresentaram melhor } \\
\text { desempenho no período da } \\
\text { tarde. ( }<<0,001) \\
\text { Independentemente do } \\
\text { método de deste } \\
\text { (individual/grupo) e traço de } \\
\text { personalidade (M/E), o } \\
\text { período da realização do teste } \\
\text { teve um efeito significativo no } \\
\text { desempenho dos adolescentes } \\
\text { (p <0,001) }\end{array}$ & $\begin{array}{l}\text { Os tipos matutinos } \\
\text { obtiveram um } \\
\text { desempenho melhor do } \\
\text { que tipos vespertinos no } \\
\text { início da manhã, onde o } \\
\text { nível de atenção e } \\
\text { desempenho do } \\
\text { vespertino } \\
\text { aumentando ao longo do } \\
\text { dia. }\end{array}$ \\
\hline $\begin{array}{l}\text { Epstein, } \\
\text { et al. } \\
1998 \\
\text { Israel }\end{array}$ & Transversal & 812 & $10-12$ & $\begin{array}{l}\text { Questionário de sono } \\
\text { Technion, } \\
\text { The Morningness- } \\
\text { Questionário noturno } \\
\text { Perguntas adicionais - } \\
\text { Sobre hábitos de sono e } \\
\text { funcionamento diurno } \\
\text { escolar. }\end{array}$ & $\begin{array}{l}\text { O grupo dos vespertinos } \\
\text { relataram maior dificuldade de } \\
\text { concentração e atenção na aula } \\
\text { e uma sonolência duas vezes } \\
\text { maior do que nos } \\
\text { intermediários (17\% vs 7\%); } \\
\text { Os matutinos relataram } \\
\text { "nunca" apresentarem } \\
\text { reclamações em sala em } \\
\text { relação a concentração e } \\
\text { sonolência comparado aos } \\
\text { intermediários (39\% vs 54\%). }\end{array}$ & $\begin{array}{l}\text { Os vespertinos } \\
\text { apresentaram } \\
\text { significativamente mais } \\
\text { queixas sobre fadiga e } \\
\text { sonolência durante os } \\
\text { dias aula. }\end{array}$ \\
\hline
\end{tabular}

*GPA (Grade Point Average): média de pontos das notas. Fonte: Autores. 


\section{Discussão}

Por ser um processo fisiológico, o sono está envolvido em diversos aspectos comportamentais como a hiperatividade, o humor e a agressividade, além de contribuir com funções cognitivas em crianças e adolescentes (Engle-Friedman et al., 2003; Zuculo et al., 2014). Durante a adolescência, o sono se torna ainda mais importante já que o indivíduo experimenta diversas transformações comuns a esta fase da vida (Lyra et al, 2017), além disso, sofre modificações, desenvolvendo características próprias e passando pela influência de hábitos de vida que geram perturbação em seu ciclo (Pinto, Pinto, Rebelo-Pinto \& Paiva 2016).

É notória a importância da identificação dos fatores que determinam os padrões de comportamento do sono na adolescência como o atraso de fase e a redução na duração de algumas de suas etapas, uma vez que, estes fatores afetam o rendimento escolar, a aprendizagem, a atenção, a memória, as relações sociais, o desenvolvimento e o estímulo cognitivo desta população, propiciando a ela maiores riscos de problemas comportamentais e psicológicos, o que afeta significativamente a qualidade de vida (Schlarb et al., 2015; Pereira et al., 2015; Franklin et al., 2018).

É importante reconhecer que os adolescentes tendem a apresentar alterações em seu ritmo circadiano devido a essas alterações de padrões e comportamento do sono, onde cronotipos vespertinos tornam-se mais prevalentes. Fato considerado um fator de risco para a saúde em uma sociedade organizada de forma diurna, onde os horários escolares e atividades rotineiras geralmente ocorrem nos turnos matutinos (Randler, 2011; Schmidt \& Randler 2010; Vollmer et al., 2017).

Nesta revisão, estudos que abordaram a temática "cronotipo, sono e aprendizagem em adolescentes" foram desenvolvidos na América, Ásia e Europa, no entanto, o grande quantitativo de estudos foi encontrado na Europa (Clarisse et al., 2010; Escribano et al., 2012; Diaz-Morales \& Escribano, 2013; Precke et al., 2013; Roeser et al., 2013; Vollmer et al., 2013; Escribano \& DIAS-MORALES, 2014; van der Vinne et al., 2015; Zerbini et al., 2017). Ressalta-se a escassez de estudos realizados no Brasil, evidenciando a necessidade desenvolver pesquisas que explorem o assunto, uma vez que que embasariam estratégias de promoção de saúde direcionadas à essa população que é tão negligenciada.

Quanto a distribuição temporal, os estudos foram desenvolvidos de 1998 até 2017, com picos em 2013 (Diaz-Morales \& Escribano, 2013; Preckel et al., 2013; Roeser et al, 2013; Vollmer et al., 2013) e 2010 (Borisenkov; Clarisse, 2010), indicando que a temática tem chamado a atenção dos pesquisadores devido a importância do sono para a qualidade de vida e consequente necessidade de conhecer os fatores que podem afetá-lo e as consequências de noite mal dormidas (Felden et al., 2016; Batista et al., 2018).

Todos os estudos incluídos nesta síntese foram transversais, que são desenhos que demonstram associação entre as variáveis e não uma relação causal (Pereira, 1995), assim, as conclusões sobre causa e efeito devem ser consideradas com cautela. Ademais, considerando que a adolescência é uma fase da vida marcada por mudanças e instabilidades biopsicossociais, um desenho longitudinal poderia fornecer informações mais concretas no que diz respeito as variáveis, além de identificar os momentos críticos presentes na passagem da infância para a adolescência.

A presente revisão encontrou associação entre o cronotipo do sono, a qualidade do sono e o rendimento escolar. Segundo os estudos de Zerbini et al. (2017) e van der Vinne et al. (2015), o cronotipo foi associado significativamente à qualidade do sono e ao rendimento escolar. Este resultado está de acordo com o trabalho de Mendes (2019), realizado com adolescentes de 12 a 17 anos, o qual concluiu que o cronotipo pode influenciar no desempenho escolar desta população.

Além disso, os resultados da revisão mostram que os adolescentes com cronotipo do sono matutino apresentam melhor rendimento escolar quando comparados aos adolescentes com cronotipo vespertino (Borisenkov et al., 2010; Clarisse et al., 2010; Escribano et al., 2012; Díaz- Morales \& Escribano, 2013; Preckel et al., 2013; Roeser et al, 2013; Vollmer et al., 2013). Este fenômeno pode ser explicado pelo estabelecimento, por parte da sociedade, do período da manhã como horário das atividades 
diárias dos adolescentes (Crowlen, Wolfson, Tarokh \& Carskadon, 2018), sendo assim, àqueles que se mostram mais despertos no turno manhã se beneficiam no tocante ao desempenho nessas atividades.

Em seus estudos Epstein, Chillag e Lavie (1998) e Escribano et al. (2014) concluíram que os adolescentes de cronotipo vespertino apresentam maior dificuldade de concentração e atenção na aula, sonolência diurna duas vezes maior do que os cronotipos intermediários e sono reduzido, respectivamente. Estes achados estão em consonância com o estudo de Gianotti et al. (2002) que encontrou associação significativa entre o cronotipo de sono, regularidade dos padrões de sono, problemas de sono, sonolência diurna e comportamento diurno visto que a prevalência de mau desempenho escolar, problemas de atenção e sonolência durante a aula foi superior em perfis vespertinos.

A explicação para estes resultados pode ser encontrada no fato da falta de sono produzir uma modulação inadequada de respostas cerebrais emocionais ao estímulo aversivo, já que adolescentes com privação do sono manifestam uma resposta hiperlímbica em decorrência da exposição a estímulos negativos. Ademais, a falta de sono também é vista como fator de peso no tocante à importante capacidade dos adolescentes em lidar com o estresse diário (Carskadon, Wolfson, Acebo, Tzischinsky \& Seifer, 1998; Gangwisch et al., 2010; Gomes et al., 2017).

Além disso, a característica do sono nesta fase da vida também pode explicar este fenômeno, visto que o sono na adolescência tende a apresentar atraso de fase e redução do seu tempo total, já que os adolescentes tendem a dormir mais tarde, porém continuam necessitando acordar cedo para exercer suas atividades diárias (Schlarb et al., 2015; Pereira et al., 2015; Pinto et al, 2016).

No que diz respeito ao nível de atenção e desempenho escolar dos adolescentes com cronotipo vespertino, o estudo de Clarisse et al. (2010) mostrou que estes adolescentes melhoram seu desempenho e nível de atenção ao longo do dia. Evidenciouse que adolescentes com o cronotipo vespertino apresentavam melhor rendimento escolar no período noturno, porém, sofriam com maior déficit de sono (Escribano \& Díaz-Morales, 2014; Zerbini et al., 2017). Como referido por Felden et al. (2016), os adolescentes que estudavam no turno da noite apresentavam maior prevalência de baixa duração de sono.

Este fato pode ser explicado pelas organizações sociais, uma vez que, geralmente, as atividades se concentram durante o dia, enquanto o repouso durante a noite, sendo assim, nos casos em que o repouso noturno é reduzido, o indivíduo tende a apresentar déficit de sono, o que pode ser expresso por meio de quadros de sonolência diurna excessiva (SDE), piores hábitos de higiene do sono, déficit de atenção e quadros de ansiedade (Gaina et al., 2006; Randler 2011; Pinto et al., 2012; Vollmer et al., 2017; Batista et al., 2018; Duarte, 2018).

Nesse sentido, ressalta-se que o cronotipo pode ser considerado um marcador biológico de desenvolvimento do adolescente, é importante considerar que o cronotipo é uma subjacente causa e não consequência, portanto torna-se importante identificar a preferência em adolescentes como fator preditor de problemas comportamentais, incluindo comportamentos de risco à saúde (LANGE, 2011).

Na presente revisão observou-se que, nos horários escolares, o cronotipo esteve associado ao nível de consciência (Preckel et al., 2013) e ao nível de atenção (Vollmer et al.; Epstein et al.; Escribano \& Díaz-Morales), em que os matutinos pareciam estar mais conscientes e ativos do que os demais. Fato que coincide com os dados encontrados no estudo Hennig et al (2017), no qual cronotipos vespertinos foram associados à maiores níveis de desatenção e baixo rendimento escolar.

Há evidências crescentes de que os ritmos sociais e adesão ao trabalho ou horários escolares têm implicações importantes e trazem um impacto significativo no sono (Rander, 2008). Além disso, sonolência e curta duração do sono podem ter um impacto negativo sobre a qualidade de vida e desenvolvimento escolar de alunos do ensino médio (KAUDERER et al., 2013). As diferenças entre os horários de acordar e dormir durante a semana e nos fins de semana também podem ter um importante impacto no bem-estar e na saúde (Tzischinsky et al. 2011). 


\section{Conclusão}

A análise dos estudos demonstrou que existe associação entre cronotipo, qualidade do sono e rendimento escolar em adolescentes, sendo o cronotipo um fator preditor do rendimento, desempenho e nível de atenção escolar.

Através das informações obtidas na presente revisão, é possível verificar uma relação entre o cronotipo, a qualidade do sono e o rendimento escolar em adolescentes, sendo o sono um fator importante a ser considerado nesta associação. Além disso, existe uma tendência a prevalência de cronotipos vespertinos nessa fase da vida, no entanto, estes se mostram associados a má qualidade do sono, menores níveis de atenção e desempenho escolar devido a presença de sonolência diurna, falta de sincronia com os horários escolares e maior risco de desenvolvimento de problemas comportamentais. Portanto, destaca-se a importância do respeito às preferências circadianas deste jovem para que seu sono não seja comprometido e, consequentemente, sua qualidade de vida seja mantida.

Torna-se então fundamental mais estudos voltados para essa temática, visando buscar alternativas para minimizar os impactos da má qualidade do sono nos adolescentes, buscando favorecer um aprendizado de qualidade, considerando o seu tempo ideal de funcionamento. Tendo em vista que ocorre essa mudança de predileção por horários mais tardios nessa fase da vida, o que tem impacto no processo de aprendizagem, ressaltamos ainda, a importância de ações voltadas a favorecer a higiene do sono, bem como orientações a estes adolescentes.

\section{Referências}

Adelantado-Renau, M., Diez-Dernandez, A., Beltran-Valls, M. R., Soriano-Maldonado, A., \& Moliner-Urdiales D. (2019). The effect of sleep quality on academic performance is mediated by Internet use time: DADOS study. Jornal de Pediatria, 95(4), 410-418.

Alvaro, P. K., Roberts, R. M., \& Harris, J. K. (2014). The independent relationships between insomnia, depression, subtypes of anxiety, and chronotype during adolescence. Sleep Medicine, 15(8),934-941.

Arbabi, T., Vollmer, C., Dörfler, T., \& Randler, C. (2014). The influence of chronotype and intelligence on academic achievement in primary school is mediated by conscientiousness, midpoint of sleep and motivation. Chronobiology International, 32(3),349-357.

Batista, G. A., Silva, T. N. D., Oliveira, M. R., Diniz, P. R. B., Lopes, S. S., \& Oliveira, L. M. F. T. (2018). Associação entre a percepção da qualidade do sono e a assimilação do conteúdo abordado em sala de aula. Revista Paulista de Pediatria, 36(3),315-321.

Bluden, S., \& Galland, B. (2014). The complexities of defining optimal sleep: Empirical and theoretical considerations with a special emphasis on children. Sleep Medicine Reviews, 18, 371-378.

Borisenkov, M. F., Perminova, E. V., \& Kosova, A. L. (2010). Chronotype, sleep length, and school achievement of 11- to 23-year-old students in northern European Russia. Chronobiology international, 27(6), 1259-1270.

Carskadon, M. A., Wolfson, A. R., Acebo, C., Tzischinsky, O., \& Seifer, R. (1998).Adolescent sleep patterns, circadian timing, and sleepiness at a transition to early school days. Sleep, 21,871-81.

Chee, K. H., Pino, N. W., \& Smith, W. L. (2005). Gender differences in the academic ethic and academic achievement. College Student Journal, $39(3)$, 604.

Clarisse, R., Le Floc'h, N., Kindelberger, C., \& Feunteun, P. (2010). Daily rhythmicity of attention in morning- vs. evening-type adolescents at boarding school under different psychosociological testing conditions. Chronobiology international, 27(4), 826-841.

Crowley, S. J., Wolfson, A. R., Tarokh, L., \& Carskadon, M. A. (2018). Na update on adolescente sleep: new evidence informing the perfect storm model. Journal of Adolescence, 67, 55-65.

Díaz-Morales, J. F., Escribano, C. (2013). Predicting school achievement: The role of inductive reasoning, sleep length and morningness-eveningness. Personality and Individual Differences, 55(2), 106-111.

Díaz-Morales, J.F., Escribano, C., \& Jankowski, K.S. (2015). Chronotype and time-of-day effects on mood during school day. Chronobiology International, $32(1), 37-42$.

Díaz-morales, J. F., \& Sánchez-lópez, M. P. (2008). Morningness-eveningness and anxiety among adults: A matter of sex/gender? Personality and Individual Differences, 44(6),1391-1401.

Epstein,R., Chillag, N., \& Lavie, P. Starting times of school: effects on daytime functioning of fifth-grade children in Israel. (1998). Sleep, 221(3),250-256.

Escribano, C., \& Díaz-Morales, J. F. (2014). Daily fluctuations in attention at school considering starting time and chronotype: an exploratory study. Chronobiology international, 31(6), 761-769. 
Escribano, C., Díaz-Morales, J. F., Delgado, P., \& Collado, M. J. (2012). Morningness/eveningness and school performance among Spanish adolescents: Further evidence. Learning and Individual Differences, 22(3), 409-413.

Duarte, L.L. (2018). Cronotipos Humanos. Cruz das Almas/BA: Editora UFRB.

Engle-Friedman, M., Riela, S., Golan, R., Ventuneac, A. M., Davis, C. M., Jefferson, A. D., \& Major, D. (2003). The effect of sleep loss on next day effort. Journal of sleep research, 12(2), 113-124.

Felden, E. P. G., Filipim, D., Barbosa, D. G., Andrade, R. D., Meyer, C., \& Louzada, F. M. (2016). Fatores associados à baixa duração duração do sono na adolescência. Revista Paulista de Pediatria, 34(1), 64-70.

Figueiredo, S., Hipolito, J., \& Tomas, C. (2019). A relação entre cronótipo e horários de desempenho cognitivo da criança Portuguesa. Psicologia, Saúde \& Doenças, 19(1), 42-49.

Franklin, A. M., Giacheti, C. M., Silva, N. C. D., Campos, L. M. G., \& Pinato, L. (2018). Correlação entre o perfil do sono e o comportamento em indivíduos com transtorno específico da aprendizagem. Communication Disorders, Audiology and Swallowing, 30(3),1-8.

Gaina, A., Sekine, M., Kanayama, H., Takashi, Y., Hu, L., Sengoku, K., \& Kagamimori, S. (2006). Morning-evening preference: Sleep pattern spectrum and lifestyle habits among Japanese junior high school pupils. Chronobiology International, 23(3), 607-621.

Gangwisch, J. E., Babiss, L. A., Malaspina, D., Turner, J. B., Zammit, G. K., \& Posner, K. (2010). Earlier parental set bedtimes as a protective factor against depression and suicidal ideation. Sleep, 33,97-106.

Giannotti, F., Cortesi, F., Sebastiani, T., \& Salvatore, O. (2002). Circadian preference, sleep and daytime behavior in adolescence, Journal of Sleep Researcha, 11,191-199.

Gomes, G. C., Passos, M. H. P. D., Silva, H. A., Oliveira, V. M. A. D., Novaes, W. A., Pitangui, A. C. R., \& Araújo, R. C. (2017). Qualidade de sono e sua associação com sintomas psicológicos em atletas adolescentes. Revista Paulista de Pediatria, 35(3), 316-321.

Hahn, C., Cowell, J. M., Wiprzycka, U. J., Goldstein, D., Ralph, M., Hasher, L., \& Zelazo, P. D. (2012). Circadian rhythms in executive function during the transition to adolescence: the effect of synchrony between chronotype and time of day. Developmental science, 15(3), 408-416.

Hairston, I. S., \& Shpitalni, R. (2016). Procrastination is linked with insomnia symptoms: The moderating role of morningness eveningness. Personality and Individual Differences, 101, 50-56.

Hennig, T., Krkovic, K., \& Lincoln, T. M. (2017). What predicts inattention in adolescents? An experience-sampling study comparing chronotype, subjective, and objective sleep parameters. Sleep medicine, 38, 58-63.

Kauderer, S., \& Randler, C. Differences in time use among chronotypes in adolescents. (2014). Biological Rhythm Research, 44(4),601-608.

Lange, L., \& Randler, C. (2011). Morningness-eveningness and behavioural problems in adolescents. Sleep and Biological Rhythms, 9, 12-18

Luciana Mello, M. D., Fernando Louzada, M. D., \& Luiz Menna-Barreto, M. D. (2001). Effects of School Schedule Transition on Sleep-Wake Cycle of Brazilian. Adolescents. Sleep and Hypnosis, 3, 3 .

Lyra, M. J., Germano-Soares, A. H., Santiago, L. C. S., Queiroz, D. R., Tassitano, R. M., Falcão, A. P. S. T., Pedrosa, R. P., Heimer, M. V., Santos, M. A. M. S. (2017). Individual and average responses of sleep quality and daytime sleepiness after four weeks of strength training in adolescents. Motriz, 23, 1-6.

Malone, S. K., Zemel, B., Compher, C., Souders, M., Chittams, J., Thompson, A. L., \& Lipman, T. H. (2016). Characteristics Associated with Sleep Duration, Chronotype, and Social Jet Lag in Adolescents. Journal of School Nursing, 32(2),120-31.

Martins, R. D. J. S. (2018). Análise do padrão de sono em estudantes do ensino médio em São Luís-MA. Dissertação de mestrado, Universidade Federal do Maranhão, São Luís, Brasil.

Mello, B. J., Mello, S. T., Vidotti, A. P., \& Mello, J. M. (2018). Cronotipo e qualidade do sono de acadêmicos do primeiro ano do curso de Medicina da cidade de Maringá-PR. Revista Saúde e Pesquisa, 11(2),287-292.

Mendes, R. A. P. D. C. (2019). Análise dos parâmetros rítmicos e de sono, cronotipo e jetlag social em adolescentes: relações com o gênero, turno escolar, atividades noturnas e desempenho cognitivo. Tese de doutorado, Universidade de São Paulo, São Paulo, SP, Brasil..

Meyer, C., Junior, G. J. F., Barbosa, D. G., Andrade,R. D., Pelegrini, A., \& Felden, E. P. G. (2017). Análise da sonolência diurna em adolescente por meio da Pediatric Daytime Sleepiness Scale: Revisão Literária. Revista Paulista de Pediatria, 35(3), 351-360.

Oliveira, W. A., Silva, L. K. T. M., Alves, S. J. O., Silva, J. G. M., \& Pinto, F. C. M. (2019). Influência da qualidade do sono sobre a aprendizagem no ensino de ciências. Revista de. Psicopedagogia, 36(109), 73-86.

Pereira, E. F., Barbosa, D. G., Andrade, R. D., Claumann, G. S., Pelegrini, A., \& Louzada, F. M. (2015) Sono e adolescência: quantas horas os adolescentes precisam dormir? Jornal Brasileiro de Psiquiatria, 64(1), 40-44.

Pereira, M. G. (1995). Epidemiologia: teoria e prática. Rio de Janeiro: Guanabara Koogan.

Pinto, T. R., Pinto, J. C., Rebelo-Pinto, H., \& Paiva, T. (2016). O sono em adolescentes portugueses: proposta de um modelo tridimensional. Análise Psicológica, 4(34), 339-352.

Preckel, F., Lipnevich, A. A., Boehme, K., Brandner, L., Georgi, K., Könen, T., Mursin, K., \& Roberts, R. D. (2013). Morningness-eveningness and educational outcomes: the lark has an advantage over the owl at high school. British Journal of Educational Psychology, 83(1),114-134. 
Pinto, T. R., Amaral, C., Silva, V. N., Silva, J., Leal, I., \& Paiva, T. (2012) Hábitos de sono e ansiedade, depressão e stresse: que relação? Actas do 12. ${ }^{\circ}$ Colóquio Internacional de Psicologia e Educação: Educação, aprendizagem e desenvolvimento: Olhares contemporâneos através da investigação e da prática. Lisboa: ISPA - Instituto Universitário, 990-1006.

Rahafar, A., Maghsdloo, M., Ferhangnia, S., \& Vollmer. (2015). The role of chronotype, gender, test anxiety, and conscientiousness in academic achievement of high school students. Chronobiology International, 33(1), 1-10.

Randler, C. (2008). Differences in sleep and circadian preference between eastern and western german adolescents. Chronobiology International, 25(4), 565575 .

Randler, C. (2011). Association between morningness-eveningness and mental and physical health in adolescents. Psychology, Health \& Medicine, 16(1), 2938 .

Roeser, K., \& Schlarb, A. (2013). The Chronotype-Academic Performance Model (CAM): Dytime Sleepiness and Learning Motivation link Chronotype and School Performance in Adolescents. Personality and Individual Differences, 54(7), 836-840.

Schlarb, A., Gulewitsch, M. D., Weltzer, V., Ellert, U., \& Enck, P. (2015). Sleep Duration and Sleep Problems in a Representative Sample of German Children and Adolescents. Health, 7(11),1397-1408.

Schmidt, S., \& Randler, C. (2010). Morningness-eveningness and eating disorders in a sample of adolescent girls. Journal of Individual Differences, 31(1), 3845 .

Thilers, P.P., MacDonald, S.W.S., \& Herlitz, A. (2006). The association between endogenous free testosterone and cognitive performance: A population-based study in 35 to 90 year-old men and women. Psychoneuroendocrinology, 31,565-76

Tzischunsky, O., \& Shochat, T. (2011). Eveningness, Sleep Patterns, Daytime Functioning, and Quality of Life in Israeli Adolescents. Chronobiology International, 28(4), 338-343

van der Vinne, V., Zerbini, G., Siersema, A., Pieper, A., Merrow, M., Hut, R. A., Roenneberg, T., \& Kantermann, T. (2015). Timing of examinations affects school performance differently in early and late chronotypes. Journal of biological rhythms, 30(1), 53-60.

Vollmer, C., Jankowski, K. S., Díaz-Morales, J. F., Itzek-Greulich, H., Wüst-Ackermann, P., \& Randler, C. (2017). Morningness-eveningness correlates with sleep time, quality, and hygiene in secondary school students: a multilevel analysis. Sleep medicine, 30, 151-159.

Wong, M. L., Lau, E. Y., Wan, J. H., Cheung, S. F., Hui, C. H., \& Mok, D. S. (2013). The interplay between sleep and mood in predicting academic functioning, physical health and psychological health: a longitudinal study. Journal of psychosomatic research, 74(4), $271-277$.

Zerbini, G., van der Vinne, V., Otto, L. K. M., Kantermann, T., Krijnen, W. P., Roenneberg, T., \& Merrow, M.. (2017). Lower school performance in late chronotypes: underlying factors and mechanisms. Scientific Reports,7(1), 4385.

Zuculo, G. M., Knap, C. C., \& Pinato, L. (2014). Correlation between sleep and quality of life in cerebral palsy. Communication Disorders, Audiology and Swallowing, 26(6),447-56. 